\title{
Chronological Changes of Quality of Life in Long-Term Survivors after Gastrectomy for Gastric Cancer
}

Wansik Yu, MD, PhD',
Ki Bum Park, MD²
Ho Young Chung, MD, PhD³
Oh Kyoung Kwon, MD2
Seung Soo Lee, MD

${ }^{1}$ Department of Surgery, Kyungpook National University School of Medicine, Daegu, ${ }^{2}$ Gastric Cancer Center, Kyungpook National University Medical Center, Daegu, ${ }^{3}$ Department of Surgery, Kyungpook National University Hospital, Daegu, Korea

\begin{abstract}
Purpose
A few studies have prospectively evaluated changes in quality of life (QoL) after surgery in short-term survivors; however, no prospective study has evaluated the longitudinal changes in QoL in long-terms survivors. We prospectively evaluated the chronological changes in QoL after a gastrectomy over a 5-year postoperative period in a large group of patients.
\end{abstract}

\section{Materials and Methods}

QoL data from the European Organization for Research and Treatment of Cancer QLQ-C30 and the QLQ-STO22 questionnaires were obtained from 254 patients who completed the entire series of QoL assessments preoperatively and at 1, 2, 3, 4, and 5 years after surgery.

\section{Results}

There was no statistically significant change in global health status/QoL during the 5-year postoperative period. Decreases in QoL from upper gastrointestinal symptoms including diarrhea $(p<0.001)$, dysphagia $(p<0.001)$, reflux symptoms $(p=0.029)$, and eating restrictions $(p<0.001)$ were observed among the long-term survivors. Decreased physical functioning $(p<0.001)$, role functioning $(p<0.001)$, and cognitive functioning $(p<0.001)$, along with fatigue $(p=0.045)$ and a poor body image $(p=0.003)$, negatively impacted the patients' QoL for a long time.

\section{Conclusion}

Management of gastrointestinal symptoms should be specifically targeted as a part of longterm patient care after a gastrectomy. Proper nutritional care will improve food intake resulting in weight gain and improved physical functioning, role functioning, and body image. In addition, patients should be encouraged to preserve self-esteem and maintain social activity.
Correspondence: Wansik Yu, MD, PhD

Gastric Cancer Center,

Kyungpook National University Medical Center,

807 Hoguk-ro, Buk-gu, Daegu 41404, Korea

Tel: 82-53-200-2700

Fax: 82-53-200-2027

E-mail:wyu@knu.ac.kr

Received October 19, 2015

Accepted December 19, 2015

Published Online January 6, 2016
Key words

Quality of life, Stomach neoplasms, Gastrectomy

\section{Introduction}

Patients with gastric cancer may have poor health-related quality of life (QoL) resulting from the disease itself before surgery. Patients may still suffer from poor QoL due to the consequences of surgery after a gastrectomy. The overall survival rate of patients with gastric cancer has increased due to the development of diagnostic tools, mass screening programs, and advancements in multimodality management.
However, significant morbidity, including early satiety, loss of appetite, heartburn, dysphagia, nausea, and vomiting is still associated with gastrectomy. These symptoms have a profound impact on QoL of postoperative patients. As a result, health providers and researchers have increasingly focused on improving life satisfaction of survivors [1].

QoL is a multi-dimensional construct covering several key dimensions including disease and treatment-related symptoms as well as physical, psychological, and social functioning. The European Organization for Research and Treatment 
of Cancer (EORTC) Quality of Life Questionnaire (QLQ)-C30 is a reliable and validated measure of the QoL of patients with cancer in international trials in oncology [2]. The Korean version of this questionnaire has also been validated [3]. The questionnaire is a cancer-specific, self-administered, structured questionnaire including 30 questions composed of both multi-item scales and single-item measures. These scales/items include five functional scales (physical, role, emotional, cognitive, and social), three symptom scales (fatigue, nausea and vomiting, and pain), a global health status/QoL scale, and six single items (dyspnea, insomnia, appetite loss, constipation, diarrhea, and financial difficulties). These 15 scales/items are grouped as follows: a global health status/QoL scale, five functional scales, and nine symptom scales/items.

The gastric cancer module (QLQ-STO22) is a supplement to the QLQ-C30 [4]. The QLQ-STO22 consists of 22 questions evaluating five multi-item symptoms scales (dysphagia, pain, reflux symptoms, eating restrictions, and anxiety), and four single items (dry mouth, taste, body image, and hair loss).

QoL has become an increasingly important factor for longterm survivors after surgery for gastric cancer. Several studies have examined QoL after a gastrectomy in patients with gastric cancer using EORTC QLQ-C30 and QLQ-STO22. A few studies have prospectively evaluated changes in QoL after surgery in short-term survivors [5-9]. However, most studies have important limitations, including, above all, small sample size [10-12] and retrospective study design $[13,14]$.

Researchers attempted to determine the relationship between postoperative QoL and the extent of gastric resection $[9,13]$, type of reconstruction after gastrectomy [15], and method of abdominal approach $[8,16]$, reaching varied conclusions [5]. As QoL changes over time, good knowledge regarding long-term chronological changes in QoL of relapse-free patients after a gastrectomy is essential to providing more appropriate care for patients. However, no study has prospectively evaluated the longitudinal changes in QoL of long-term survivors.

In this study, we prospectively evaluated the chronological changes in QoL after a gastrectomy over a 5-year postoperative period in a large group of patients.

\section{Materials and Methods}

\section{Patients}

A total of 1,105 patients who underwent a curative gastrec- tomy for primary gastric cancer between January 2008 and June 2010 at the Kyungpook National University Hospital were enrolled in this prospective cohort study. Among them, 439 patients completed the entire series of QoL assessments during the 5-year postoperative period; 161 patients with co-morbidities that could affect the QoL including cardiovascular disease, diabetes mellitus, chronic respiratory disease, chronic hepatic disease, and cerebrovascular disease and 24 patients who underwent another operation were excluded. The QoL data obtained from the remaining 254 patients were analyzed. The study was approved by the Institutional Review Board of Kyungpook National University Hospital (No. 201510035001).

The demographic characteristics of the patients are shown in Table 1. There were 95 female and 159 male patients. The mean age was $54.9 \pm 10.7$ years. Most patients had stage I disease. Subtotal gastrectomy was performed on 202 patients and 52 patients underwent a total gastrectomy. Patients showed no evidence of recurrence at the final follow-up visit.

Table 1. Characteristics of patients

\begin{tabular}{|cc}
\hline Characteristic & No. of patients $(\%)$ \\
\hline Age, mean \pm SD (yr) & $54.9 \pm 10.7$ \\
Sex & \\
Female & $95(37.4)$ \\
\hline Male & $159(62.6)$ \\
\hline Stage ${ }^{a)}$ & \\
\hline IA & $171(67.3)$ \\
IB & $30(11.8)$ \\
\hline IIA & $23(9.1)$ \\
IIB & $15(5.9)$ \\
IIIA & $8(3.1)$ \\
IIIB & $5(2.0)$ \\
\hline IIIC & $1(0.4)$ \\
\hline IV & $1(0.4)$ \\
Pathological type & \\
\hline Differentiated & $97(38.2)$ \\
\hline Undifferentiated & $157(61.8)$ \\
\hline Type of gastrectomy & \\
\hline Open distal gastrectomy & $161(63.4)$ \\
\hline Open total gastrectomy & $48(18.9)$ \\
\hline Laparoscopy-assisted distal gastrectomy & $41(16.1)$ \\
\hline Laparoscopy-assisted total gastrectomy & $3(1.2)$ \\
\hline Robot-assisted total gastrectomy & $1(0.4)$ \\
\hline Adjuvant chemotherapy & \\
\hline Yes & $30(11.8)$ \\
\hline No & $224(88.2)$ \\
\hline
\end{tabular}

a)The 7th edition of the American Joint Committee on Cancer Classification. 
Table 2. Mean scores of EORTC QLQ-C30 and QLQ-STO22 scales/items

\begin{tabular}{|c|c|c|c|}
\hline Variable & Baseline & 5 Years after a gastrectomy & p-value \\
\hline Global health status/QoL & $67.7 \pm 21.4$ & $73.5 \pm 23.5$ & 0.101 \\
\hline \multicolumn{4}{|l|}{ QLQ-C30 function scales } \\
\hline Physical functioning & $91.4 \pm 9.7$ & $85.6 \pm 14.3$ & $<0.001$ \\
\hline Role functioning & $93.2 \pm 14.3$ & $86.7 \pm 19.8$ & $<0.001$ \\
\hline Emotional functioning & $80.0 \pm 19.3$ & $85.5 \pm 18.2$ & 0.012 \\
\hline Cognitive functioning & $90.8 \pm 13.5$ & $84.2 \pm 15.8$ & $<0.001$ \\
\hline Social functioning & $88.2 \pm 18.0$ & $87.4 \pm 18.8$ & $>0.999$ \\
\hline \multicolumn{4}{|c|}{ QLQ-C30 symptom scales/items } \\
\hline Fatigue & $19.3 \pm 18.9$ & $24.4 \pm 19.8$ & 0.045 \\
\hline Nausea and vomiting & $6.7 \pm 13.8$ & $9.9 \pm 15.2$ & 0.177 \\
\hline Pain & $8.9 \pm 15.7$ & $8.1 \pm 13.8$ & $>0.999$ \\
\hline Dyspnea & $8.3 \pm 16.4$ & $12.5 \pm 19.6$ & 0.128 \\
\hline Insomnia & $13.3 \pm 22.5$ & $14.2 \pm 24.3$ & $>0.999$ \\
\hline Appetite loss & $8.8 \pm 18.9$ & $10.4 \pm 20.4$ & $>0.999$ \\
\hline Constipation & $11.2 \pm 18.8$ & $13.1 \pm 20.2$ & 0.988 \\
\hline Diarrhea & $11.4 \pm 19.7$ & $21.0 \pm 23.4$ & $<0.001$ \\
\hline Financial difficulties & $16.6 \pm 25.7$ & $14.3 \pm 22.8$ & 0.993 \\
\hline \multicolumn{4}{|c|}{ QLQ-STO22 symptom scales/items } \\
\hline Dysphagia & $4.6 \pm 8.9$ & $9.3 \pm 11.1$ & $<0.001$ \\
\hline Pain & $13.6 \pm 15.3$ & $13.8 \pm 15.1$ & $>0.999$ \\
\hline Reflux symptoms & $9.7 \pm 14.5$ & $13.9 \pm 16.2$ & 0.029 \\
\hline Eating restrictions & $5.3 \pm 10.2$ & $12.4 \pm 14.8$ & $<0.001$ \\
\hline Anxiety & $23.9 \pm 20.6$ & $25.7 \pm 20.0$ & $>0.999$ \\
\hline Dry mouth & $15.5 \pm 22.8$ & $20.1 \pm 25.4$ & 0.661 \\
\hline Taste & $3.3 \pm 11.6$ & $6.2 \pm 15.2$ & 0.232 \\
\hline Body image & $13.6 \pm 23.4$ & $21.8 \pm 26.1$ & 0.003 \\
\hline Hair loss & $34.7 \pm 35.3$ & $38.2 \pm 28.3$ & $>0.999$ \\
\hline
\end{tabular}

Values are presented as mean \pm standard deviation. EORTC, European Organization for Research and Treatment of Cancer; QLQ, Quality of Life Questionnaire; QoL, quality of life.

\section{QoL assessment}

QoL was assessed using the Korean Versions of the EORTC QLQ-C30 and QLQ-STO22. All patients were asked to complete the questionnaires by themselves on the day of admission and at regular follow-up visits $(1,2,3,4$, and 5 years after surgery). The raw scores were transformed to scores ranging from 0 to 100 according to the scoring manual provided by the EORTC [17]. A high score for a functional scale represents a high/healthy level of functioning and a high score for the global health status / QoL represents a high QoL, but a high score for a symptom scale/item represents a high level of symptomatology/problems. For the EORTC QLQ-STO22, a high score represents a low QoL.

\section{Statistical analyses}

The mean scores of each scale were compared in a longi- tudinal fashion across five postoperative years. One way analysis of variance followed by a Bonferroni post-hoc test or Dunnett T3 post-hoc test was performed for comparison of differences in the mean scores among the time intervals. p-values of less than 0.05 were considered statistically significant.

\section{Results}

Preoperative and 5-year postoperative mean scores of EORTC QLQ-C30 and QLQ-STO22 scales/items are shown in Table 2.

Global health status / QoL tended to increase after the first postoperative year and the mean score at the 5th postoperative year was significantly higher than 1 year after the gas- 


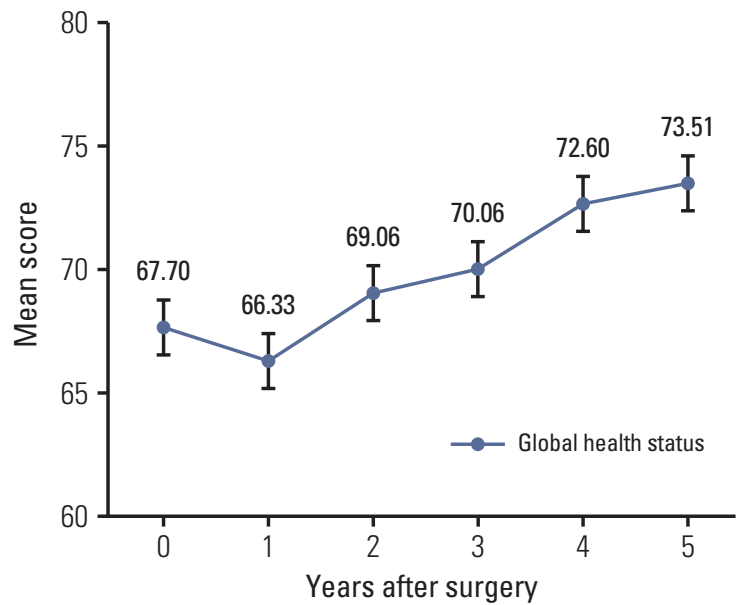

Fig. 1. Mean scores and 95\% confidence intervals of global health status / QoL scale of EORTC QLQ-C30 at each time point. QoL, quality of life; EORTC, European Organization for Research and Treatment of Cancer; QLQ, Quality of Life Questionnaire.

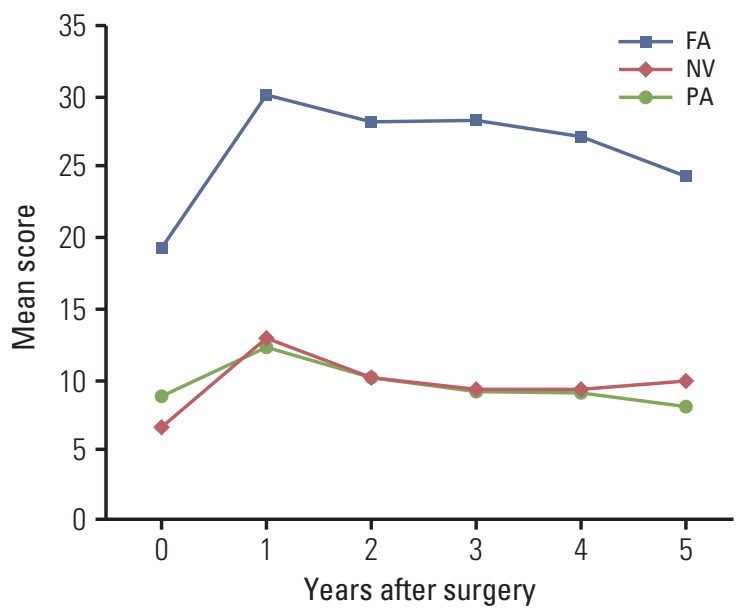

Fig. 3. Mean scores of symptom scales of EORTC QLQC30 at each time point. EORTC, European Organization for Research and Treatment of Cancer; QLQ, Quality of Life Questionnaire; FA, fatigue; NV, nausea and vomiting; PA, pain.

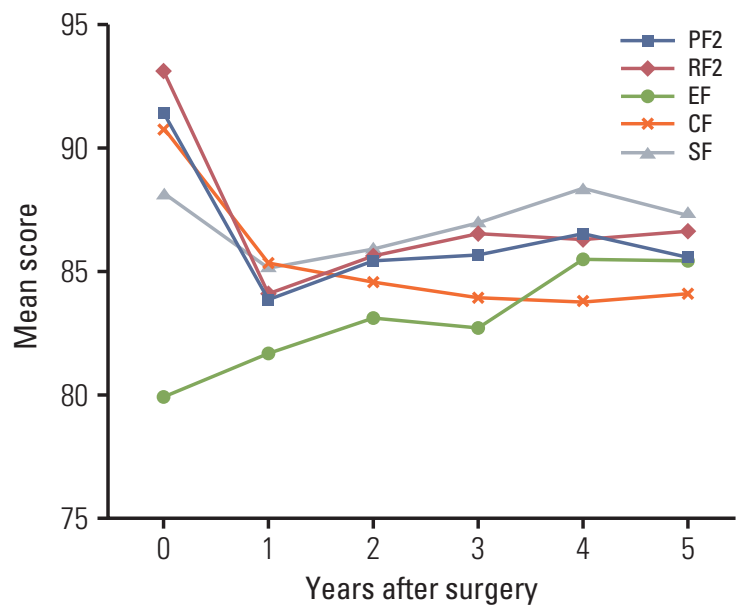

Fig. 2. Mean scores of functional scales of EORTC QLQC30 at each time point. EORTC, European Organization for Research and Treatment of Cancer; QLQ, Quality of Life Questionnaire; PF2, physical functioning; RF2, role functioning; $\mathrm{EF}$, emotional functioning; $\mathrm{CF}$, cognitive functioning; $\mathrm{SF}$, social functioning.

trectomy ( $\mathrm{p}=0.012$ ) (Fig. 1). Physical functioning score, role functioning score, and cognitive functioning score decreased significantly in the first postoperative year $(\mathrm{p}<0.001$, $\mathrm{p}<0.001$, and $\mathrm{p}=0.001$, respectively), but showed no significant changes thereafter. Emotional functioning was worst at baseline and improved steadily after surgery. Social functioning scores showed no statistically significant changes compared to preoperative score during the 5 -year postoperative period (Fig. 2).

Fatigue score increased significantly 1 year after surgery $(p<0.001)$ and then decreased gradually. However, scores remained high during the 5 years postoperatively $(\mathrm{p}=0.045)$. Nausea and vomiting score and pain score increased 1 year after surgery without significance and tended to decrease afterward (Fig. 3).

There were no statistically significant changes in dyspnea, insomnia, appetite loss, and constipation scores compared with preoperative scores during the 5-year postoperative period. Financial difficulties showed a similar pattern. Diarrhea score increased significantly 1 year after surgery $(p<0.001)$. Although there was a tendency to decrease afterward, diarrhea score was significantly higher 5 years after surgery compared with the preoperative level (Fig. 4).

The changes in the mean scores of QLQ-STO22 scales/ items are shown in Figs. 5 and 6. Dysphagia and eating restrictions showed significant worsening 1 year after surgery $(p<0.001$ and $p<0.001$, respectively). Despite gradual improvements in these symptoms, the scores showed signif- 


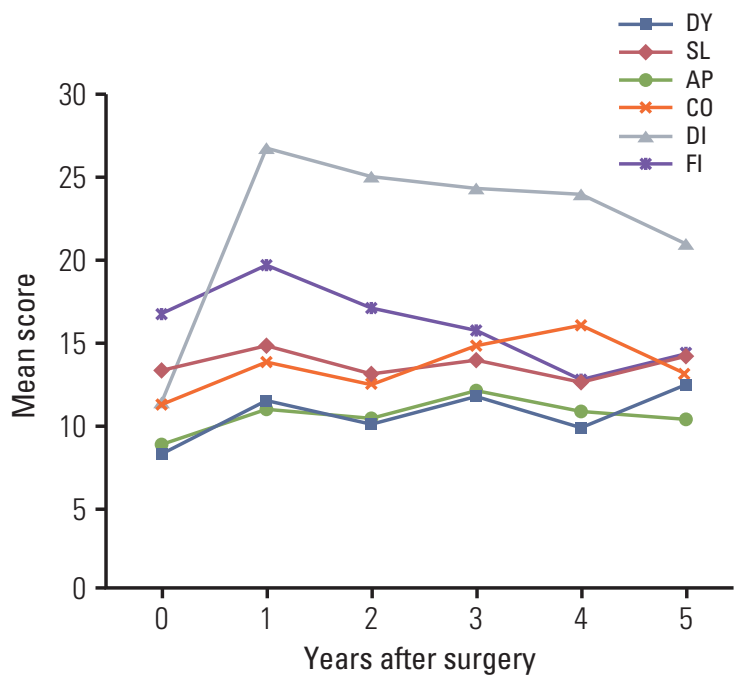

Fig. 4. Mean scores of single items of EORTC QLQ-C30 at each time point. EORTC, European Organization for Research and Treatment of Cancer; QLQ, Quality of Life Questionnaire; DY, dyspnea; SL, insomnia; AP, appetite loss; CO, constipation; DI, diarrhea; FI, financial difficulties.

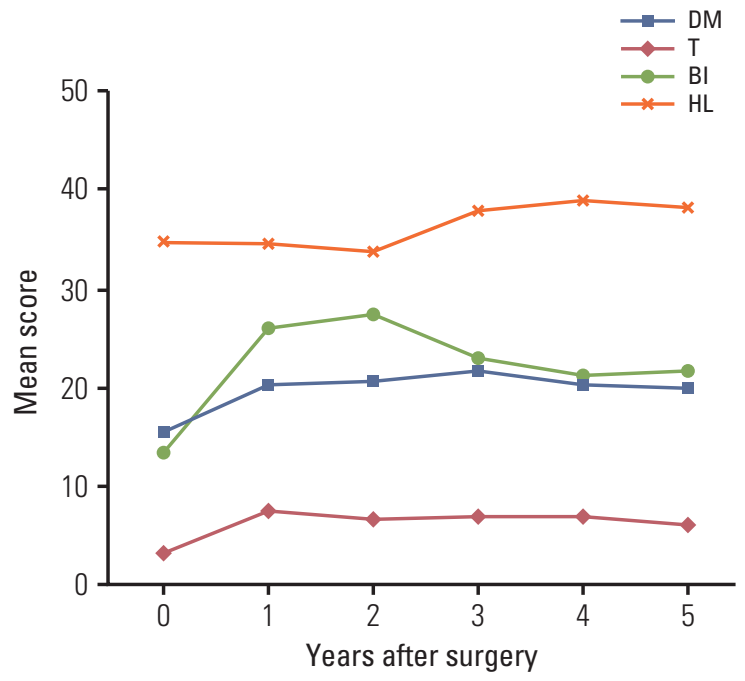

Fig. 6. Mean scores of single items of EORTC QLQ-STO22 at each time point. EORTC, European Organization for Research and Treatment of Cancer; QLQ, Quality of Life Questionnaire; DM, dry mouth; T, taste; BI, body image; $\mathrm{HL}$, hair loss.

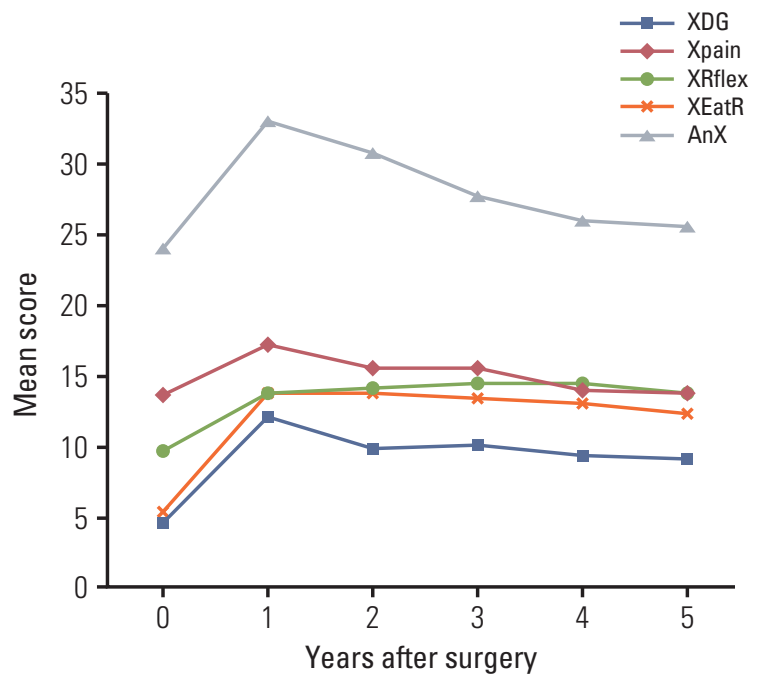

Fig. 5. Mean scores of scales of EORTC QLQ-STO22 at each time point. EORTC, European Organization for Research and Treatment of Cancer; QLQ, Quality of Life Questionnaire; XDG, dysphagia; XPain, pain; XRflex, reflux symptoms; XEatR, eating restrictions; Anx, anxiety.

icantly higher levels compared with preoperative levels 5 years after the gastrectomy ( $\mathrm{p}<0.001$ and $\mathrm{p}<0.001$, respectively). Reflux symptoms persisted during the 5-year postoperative period without significant changes. Anxiety had increased significantly 1 year after surgery $(p<0.001)$, then decreased gradually. The difference showed no statistical significance 3 years after a gastrectomy. Although taste score showed significant worsening 1 year after surgery $(p=0.008)$, there were no significant differences after 2 years postoperatively. Worsened body image had not recovered at 5 years after the gastrectomy. No significant changes were observed for pain, dry mouth, and hair loss.

\section{Discussion}

Similar to another study [5], our results showed that the global health status/QoL decreased slightly during the first postoperative year and tended to increase afterward. Social functioning, nausea and vomiting, and pain showed a similar pattern. There were no statistically significant changes in the dyspnea, insomnia, appetite loss, constipation, financial difficulties, dry mouth, and hair loss scores compared with preoperative scores during the 5-year postoperative period. Hair loss is often used for assessment of QoL related to chem- 
otherapy or radiotherapy for various types of cancer. Former cancer patients appear to pay less attention to their hair as long as they remain free of disease following cancer surgery. Similar to other studies $[7,11,12]$, emotional functioning was the worst at baseline and showed constant improvement after surgery. Emotional functioning appears to be related to the depression caused by the initial diagnosis of cancer, and patients were gradually relieved from depression regarding their disease over time. Anxiety score also reached preoperative level 5 years after the gastrectomy.

A gastrectomy has a temporary negative impact on most aspects of QoL, and recovery typically occurs within the six or 12 postoperative months $[5,10]$. However, some aspects of impaired QoL persist for a long period. The QoL of long-term survivors after a gastrectomy is influenced by the surgery itself as well as past memories of malignancy even when patients are considered to be disease-free [18].

Kong et al. [7] reported that physical functioning and role functioning showed the lowest scores after surgery and did not fully recover to preoperative levels until 1 year after surgery. In addition, scores for fatigue, nausea and vomiting, diarrhea, dysphagia, eating restriction, anxiety, taste, and body image remained significantly higher 1 year after surgery. Therefore, the authors recommended further followup to confirm whether these scales/items will recover or not. Among them, our study showed that scores for nausea and vomiting, anxiety, and taste gradually returned to the preoperative levels and showed no significant differences 5 years after a gastrectomy. However, changed physical functioning, role functioning, fatigue, diarrhea, dysphagia, eating restrictions, and body image did not recover even 5 years after a gastrectomy.

Cancer and its treatments are associated with increased fatigue that may persist for years after cancer eradication [19]. Our results showed that fatigue score was the highest 1 year after the gastrectomy and remained at a significantly increased level until 5 years after surgery.

Although there was no statistically significant change in global health status / QoL, our study found low QoL related to upper gastrointestinal symptoms including diarrhea, dysphagia, reflux symptoms, and eating restrictions in longterm survivors. Decreased physical functioning, role functioning, and cognitive functioning, fatigue, and a poor body image also impact QoL for a long time.

While surviving 5 years after surgery is often regarded as a turning point in the fight against cancer, in terms of QoL, survivors after a gastrectomy still have persistent needs for management of QoL $[18,20]$. Management of symptoms should be specifically targeted as part of patient care after a gastrectomy. Development of appropriate interventions and individual management for each symptom by surgeons is required for patients with these symptoms.
The ultimate goal of the QoL assessment of cancer patients is to restore them to a healthy period as rapidly as possible [18]. Although physical functioning, role functioning, cognitive functioning, and body image may deteriorate with age, proper nutritional care including monitoring and correcting patients' daily dietary habits will improve food intake resulting in weight gain and improved physical functioning, role functioning, and body image [6]. Patients with impaired cognitive functioning should undergo a complete medical checkup and consult with a neurologist or a psychiatrist. In addition, patients should be encouraged to preserve selfesteem and maintain social activity [18]. These suggestions can be applied to relapse-free survivors after gastrectomy.

It is recommended that changes in postoperative QoL be used to inform patients about what to expect after surgery and to guide timely supportive postoperative interventions. Surgeons should reassure patients that QoL will mostly return to baseline about 1 year after surgery and that QoL related to upper gastrointestinal symptoms will still be lower but these symptoms will be managed individually and on a timely basis.

This study has several strengths. We enrolled consecutive patients undergoing gastrectomy by the same small group of surgeons at one institution and prospectively collected data on QoL. The inclusion of the preoperative QoL data of all patients and the postoperative assessments at fixed times enables the comparison of QoL between baseline and postoperative changes in QoL. However, premorbid QoL may be the most valid baseline measure of QoL. Preoperative QoL refers to QoL that has not yet been altered by surgery and still represents postmorbid QoL. Preoperative-based interpretations of postoperative change in QoL may not only exaggerate the persistence of decreased QoL but also conceal ongoing QoL deterioration after surgery [1]. Despite this limitation, we considered preoperative QoL as the baseline because acquiring data regarding premorbid QoL from patients was not feasible in clinical practice.

\section{Conclusion}

Management of gastrointestinal symptoms should be specifically targeted as a part of long-term patient care after a gastrectomy. Proper nutritional care will improve food intake resulting in weight gain and improved physical functioning, role functioning, and body image. In addition, patients should be encouraged to preserve self-esteem and maintain social activity. 


\section{Conflicts of Interest}

Conflict of interest relevant to this article was not reported.

\section{Acknowledgments}

This research was supported by the Kyungpook National University Research Fund, 2012.

\section{References}

1. Lee SS, Chung HY, Kwon O, Yu W. Long-term shifting patterns in quality of life after distal subtotal gastrectomy: preoperative- and healthy-based interpretations. Ann Surg. 2015; 261:1131-7.

2. Aaronson NK, Ahmedzai S, Bergman B, Bullinger M, Cull A, Duez NJ, et al. The European Organization for Research and Treatment of Cancer QLQ-C30: a quality-of-life instrument for use in international clinical trials in oncology. J Natl Cancer Inst. 1993;85:365-76.

3. Yun YH, Park YS, Lee ES, Bang SM, Heo DS, Park SY, et al. Validation of the Korean version of the EORTC QLQ-C30. Qual Life Res. 2004;13:863-8.

4. Vickery CW, Blazeby JM, Conroy T, Arraras J, Sezer O, Koller $\mathrm{M}$, et al. Development of an EORTC disease-specific quality of life module for use in patients with gastric cancer. Eur J Cancer. 2001;37:966-71.

5. Karanicolas PJ, Graham D, Gonen M, Strong VE, Brennan MF, Coit DG. Quality of life after gastrectomy for adenocarcinoma: a prospective cohort study. Ann Surg. 2013;257:1039-46.

6. Kim AR, Cho J, Hsu YJ, Choi MG, Noh JH, Sohn TS, et al. Changes of quality of life in gastric cancer patients after curative resection: a longitudinal cohort study in Korea. Ann Surg. 2012;256:1008-13.

7. Kong H, Kwon OK, Yu W. Changes of quality of life after gastric cancer surgery. J Gastric Cancer. 2012;12:194-200.

8. Misawa K, Fujiwara M, Ando M, Ito S, Mochizuki Y, Ito Y, et al. Long-term quality of life after laparoscopic distal gastrectomy for early gastric cancer: results of a prospective multiinstitutional comparative trial. Gastric Cancer. 2015;18:417-25.

9. Park S, Chung HY, Lee SS, Kwon O, Yu W. Serial comparisons of quality of life after distal subtotal or total gastrectomy: what are the rational approaches for quality of life management? J Gastric Cancer. 2014;14:32-8.

10. Avery K, Hughes R, McNair A, Alderson D, Barham P, Blazeby J. Health-related quality of life and survival in the 2 years after surgery for gastric cancer. Eur J Surg Oncol. 2010;
36:148-54.

11. Kobayashi D, Kodera Y, Fujiwara M, Koike M, Nakayama G, Nakao A. Assessment of quality of life after gastrectomy using EORTC QLQ-C30 and STO22. World J Surg. 2011;35:357-64.

12. Park JY, Eom BW, Jo MJ, Yoon HM, Ryu KW, Kim YW, et al. Health-related quality of life after robot-assisted distal gastrectomy in early gastric cancer. World J Surg. 2014;38:1112-20.

13. Bae JM, Kim S, Kim YW, Ryu KW, Lee JH, Noh JH, et al. Health-related quality of life among disease-free stomach cancer survivors in Korea. Qual Life Res. 2006;15:1587-96.

14. Huang CC, Lien HH, Wang PC, Yang JC, Cheng CY, Huang CS. Quality of life in disease-free gastric adenocarcinoma survivors: impacts of clinical stages and reconstructive surgical procedures. Dig Surg. 2007;24:59-65.

15. Namikawa T, Oki T, Kitagawa H, Okabayashi T, Kobayashi M, Hanazaki K. Impact of jejunal pouch interposition reconstruction after proximal gastrectomy for early gastric cancer on quality of life: short- and long-term consequences. Am J Surg. 2012;204:203-9.

16. Kim YW, Baik YH, Yun YH, Nam BH, Kim DH, Choi IJ, et al. Improved quality of life outcomes after laparoscopy-assisted distal gastrectomy for early gastric cancer: results of a prospective randomized clinical trial. Ann Surg. 2008;248:721-7.

17. Fayers PM, Aaronson NK, Bjordal K, Groenvold M, Curran D, Bottomley A, et al. The EORTC QLQ-C30 scoring manual. 3rd ed. Brussels: European Organisation for Research and Treatment of Cancer; 2001.

18. Lee SS, Chung HY, Kwon OK, Yu W. Quality of life in cancer survivors 5 years or more after total gastrectomy: a case-control study. Int J Surg. 2014;12:700-5.

19. Stone P, Richards M, Hardy J. Fatigue in patients with cancer. Eur J Cancer. 1998;34:1670-6.

20. Lee SS, Chung HY, Yu W. Quality of life of long-term survivors after a distal subtotal gastrectomy. Cancer Res Treat. 2010;42:130-4. 